Supporting Information for

\title{
A Crossed Molecular Beams and Computational Study on the Formation of the Astronomically Elusive Thiosilaformyl Radical (HSiS, $\mathbf{X}^{2} \mathbf{A}^{\prime}$ )
}

Shane J. Goettl ${ }^{\mathrm{a}}$, Zhenghai Yanga, Srinivas Doddipatla ${ }^{\mathrm{a}}$, Chao Heª Márcio O. Alves $^{\mathrm{b}}$, Breno R. L.

$$
\text { Galvão }^{\mathrm{b}, *} \text {, Ralf I. Kaiser }{ }^{\mathrm{a}, *}
$$

${ }^{a}$ Department of Chemistry, University of Hawai ‘i at Mānoa, Honolulu, HI 96822, USA.

*Corresponding Author Prof. Ralf I. Kaiser: ralfk@hawaii.edu

${ }^{b}$ Centro Federal de Educação Tecnológica de Minas Gerais, CEFET-MG, Av. Amazonas 5253, 30421-169 Belo Horizonte, Minas Gerais, Brazil

*Corresponding Author Prof. Breno R L Galvão: brenogalvao@gmail.com 


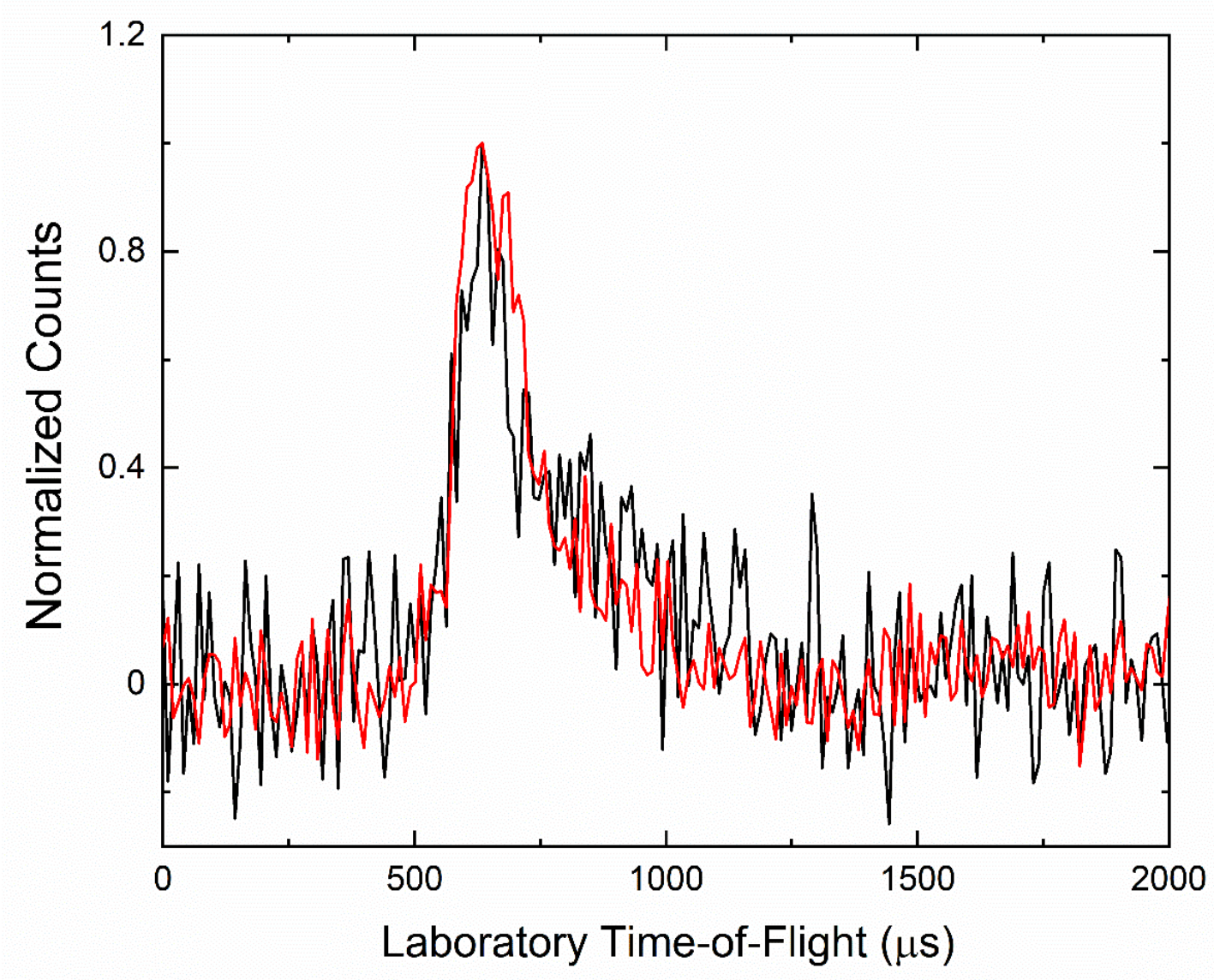

Figure S1. Time-of-flight (TOF) spectra taken at the center-of-mass (CM) angle of $54^{\circ}$ corresponding to $\mathrm{m} / \mathrm{z}=60$ (black) and $\mathrm{m} / \mathrm{z}=61$ (red) for the reaction of atomic silicon $\left(\operatorname{Si}\left({ }^{3} \mathrm{P}_{\mathrm{j}}\right)\right.$ ) with thiomethanol $\left(\mathrm{CH}_{3} \mathrm{SH}\right)$. 


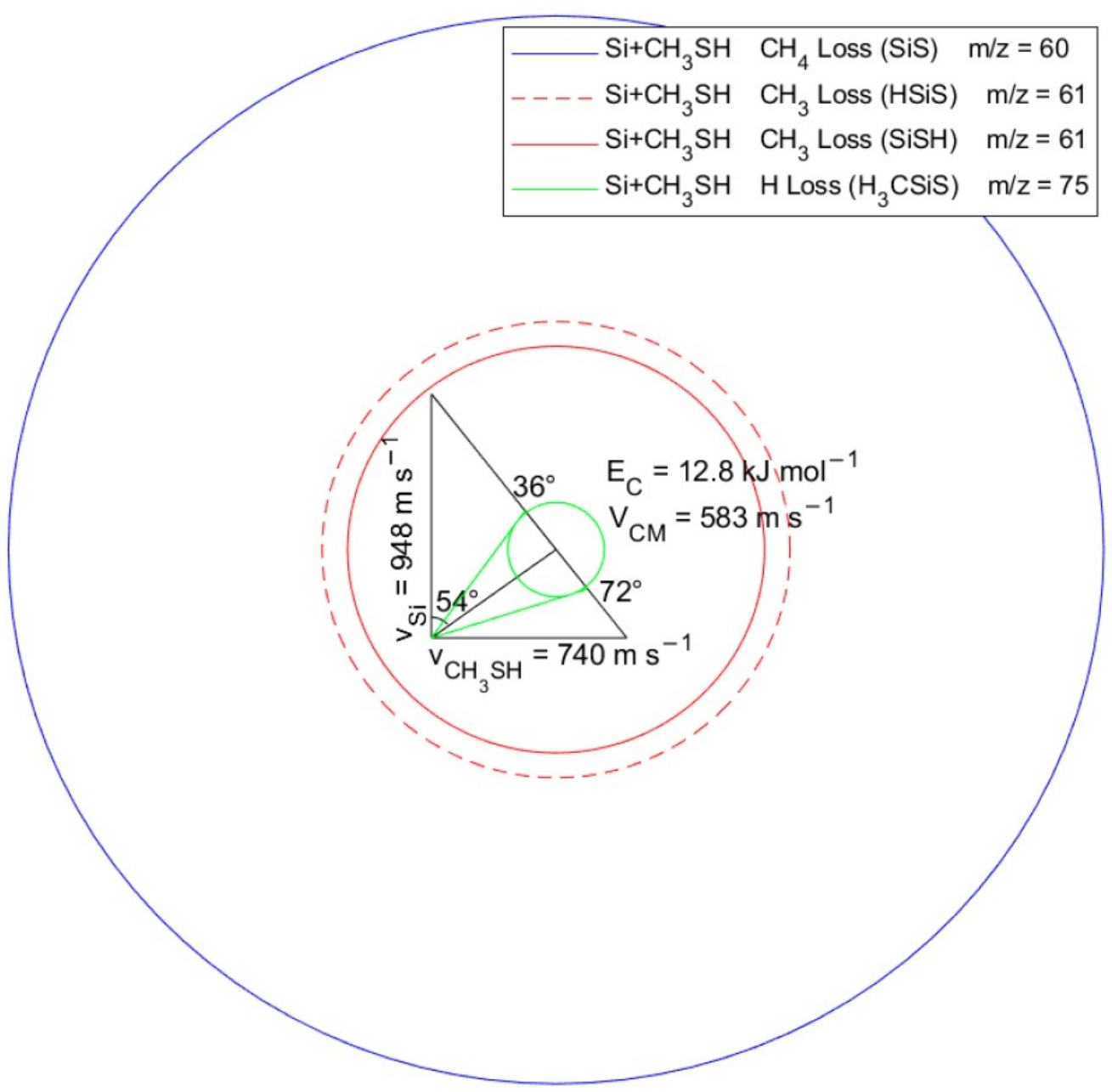

Figure S2. Newton circles for the reaction of atomic silicon $\left(\mathrm{Si}\left({ }^{3} \mathrm{P}_{\mathrm{j}}\right)\right)$ with thiomethanol $\left(\mathrm{CH}_{3} \mathrm{SH}\right)$ for the methane and methyl loss channels along with the atomic hydrogen emission pathway. Each Newton circle has a radius equal to the maximum CM recoil velocity of the heavy product. $E_{c}$ and $V_{C M}$ define the collision energy and velocity of the center of mass, respectively. 


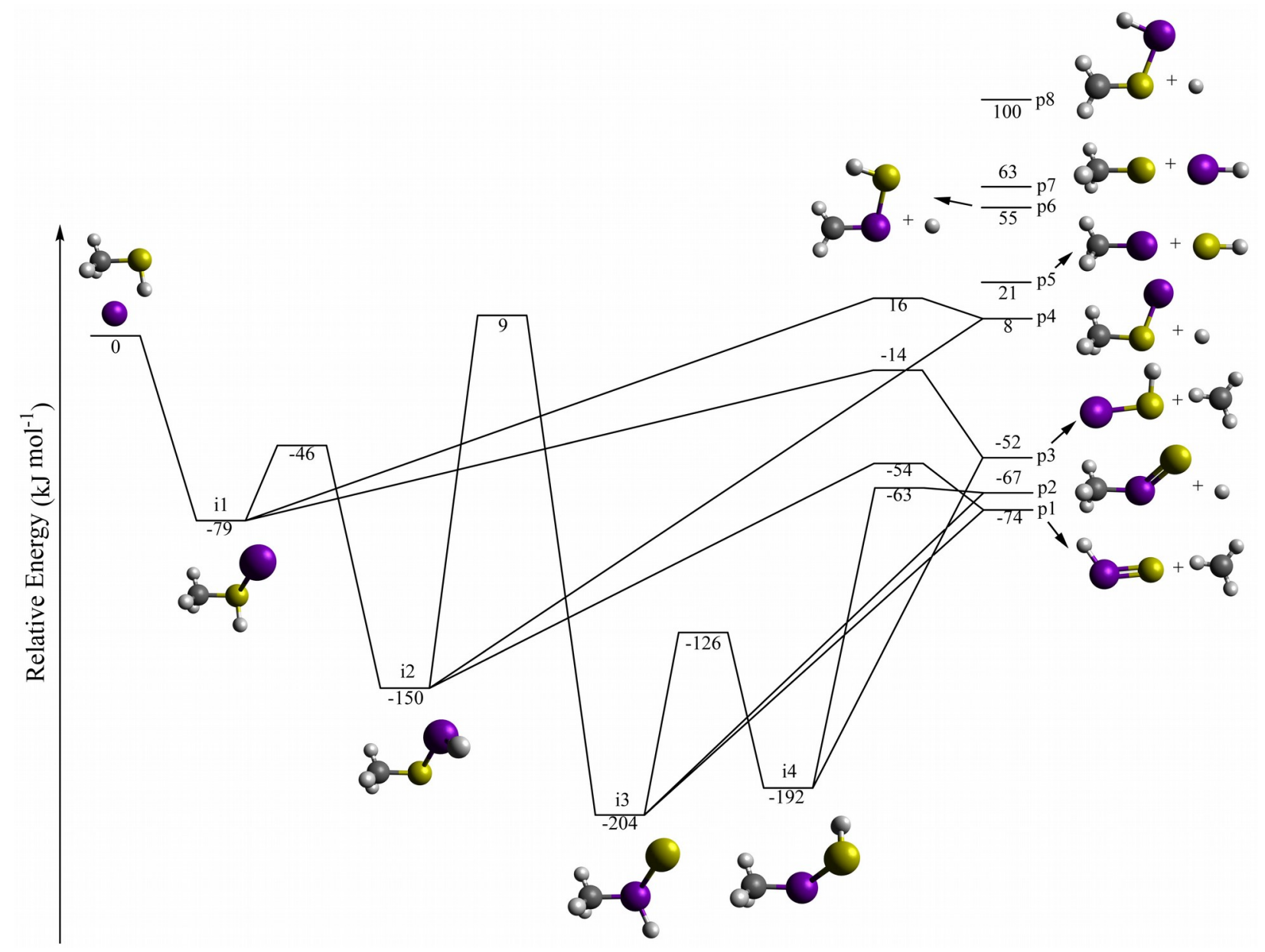

Fig. S3: Complete represenation of the calculated products, intermediates, and transition states for the reaction between ground state atomic silicon and thiomethanol calculated at the CCSD(T)-F12/aug-cc-pV(T+d)Z//M06-2X/cc-pV(T+d)Z+ZPE(M06-2X/cc-pV(T+d)Z) level. Atoms are colored as follows: carbon (grey); silicon (purple); hydrogen (white); sulfur (yellow). 
Table S1. Energy-dependent rate constants derived from RRKM calculations.

\begin{tabular}{cc}
\hline Reaction Pathway & Rate Constant $\left(\mathrm{s}^{-1}\right)$ \\
\hline $\mathrm{i} 1 \rightarrow \mathrm{i} 2$ & $5.35267 \times 10^{11}$ \\
$\mathrm{i} 2 \rightarrow \mathrm{i} 1$ & $2.41159 \times 10^{9}$ \\
$\mathrm{i} 1 \rightarrow \mathrm{p} 3$ & $1.13573 \times 10^{10}$ \\
$\mathrm{i} 2 \rightarrow \mathrm{p} 4$ & $1.08041 \times 10^{11}$ \\
\hline
\end{tabular}


Table S2. Optimized Cartesian coordinates (Angstrom) and vibrational frequencies $\left(\mathrm{cm}^{-1}\right)$ for all intermediates, transition states, reactants, and products involved in the reactions of atomic silicon $\left(\mathrm{Si}\left({ }^{3} \mathrm{P}_{\mathrm{j}}\right)\right)$ with thiomethanol $\left(\mathrm{CH}_{3} \mathrm{SH}\right)$ at the M06-2X/cc-pV(T+d)Z level.

\section{Reagents}

\section{$\mathrm{CH}_{3} \mathrm{SH}$}

$\begin{array}{lllc}\mathrm{C} & -3.41176033 & 1.25439584 & 0.01449201 \\ \mathrm{~S} & -1.60045278 & 1.28984702 & 0.02218826 \\ \mathrm{H} & -3.82207370 & 1.90858257 & 0.77812487 \\ \mathrm{H} & -3.78685069 & 0.24311264 & 0.14125393 \\ \mathrm{H} & -3.71781158 & 1.62130284 & -0.96190315 \\ \mathrm{H} & -1.43594086 & 0.82071924 & 1.26236403\end{array}$

Frequencies

236.041

732.010

804.927

977.459

1101.351

1361.538

1481.980

1494.617

2741.296

3089.114

3176.547

3177.949

\section{Products}

\section{$\mathrm{CH}_{3}$}

$\begin{array}{rrrr}\mathrm{C} & 0.66410094 & 2.73329639 & -0.00000000 \\ \mathrm{H} & 1.51476645 & 2.07351351 & 0.00000000 \\ \mathrm{H} & 0.81006140 & 3.79990077 & 0.00000000 \\ \mathrm{H} & -0.33263868 & 2.32650924 & 0.00000000\end{array}$

Frequencies

405.863

1412.233 
1412.371

3144.143

3323.125

3323.298

\section{HSiS}

S -3.5140755200 1.2168792500 0.0000000000

Si -1.5816367900 1.4356775300 0.0000000000

H - -0.69533771000 .22320321000 .0000000000$

Frequencies

580.731

724.768

2077.406

\section{HSSi}

$$
\begin{array}{lrrr}
\text { Si } & -6.0232639300 & -0.4069621600 & -0.0000000000 \\
\text { S } & -4.1160321200 & 0.4944951200 & -0.0000000000 \\
\text { H } & -3.3237240300 & -0.5920630100 & 0.0000000000
\end{array}
$$

Frequencies

518.854

675.323

2651.064

\section{$\mathrm{CH}_{3} \mathrm{~S}$}

$$
\begin{array}{lrrr}
\mathrm{C} & -3.4018590482 & 1.2522998432 & 0.0201163997 \\
\mathrm{~S} & -1.6137606348 & 1.2888270144 & 0.0216427185 \\
\mathrm{H} & -3.8166823624 & 1.9128767148 & 0.7775576358 \\
\mathrm{H} & -3.7817406913 & 0.2403778458 & 0.1372127499 \\
\mathrm{H} & -3.7171155122 & 1.6208224788 & -0.9593757732
\end{array}
$$

Frequencies

633.337

743.794

869.142

1337.308

1387.002

1478.986 
3053.926

3129.352

3153.624

\section{$\mathrm{CH}_{3} \mathrm{Si}$}

$\begin{array}{llll}\text { C } & -3.4070510575 & 1.2427420340 & 0.0450102109\end{array}$

Si $\quad-1.5211101617 \quad 1.3041677327-0.0129868177$

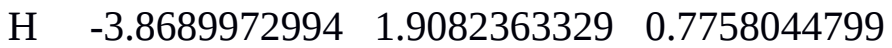

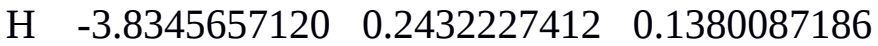

H $\quad-3.69943401831 .6168350561-0.9486828610$

Frequencies

502.819

621.844

693.285

1242.984

1363.488

1444.282

2996.870

3086.121

3124.474

$\mathrm{SiH}$

Si $\quad-1.4471899572 \quad 1.5322851907 \quad 0.1340533318$

H $\quad-0.7836439977 \quad 0.1846410261 \quad-0.0654881498$

Frequencies

2060.131

SH

S $\quad-4.0806275850 \quad 0.5482255800 \quad 0.1585125495$

$\begin{array}{llll}\mathrm{H} & -3.3925807519 & -0.5980317347 & 0.2394575334\end{array}$

Frequencies

2727.726

\section{$\mathrm{H}_{3} \mathrm{CSiS}$}

Si $-1.2922444017 \quad 2.3447081197 \quad-0.9116030215$ 
$\begin{array}{llll}\text { C } & 0.3530548631 & 2.5341783821 & -0.0094074653\end{array}$

$\begin{array}{lllll}\text { S } & -3.0086234334 & 2.7190429318 & -0.0681228236\end{array}$

H $\quad 0.3988512280 \quad 3.5383974752 \quad 0.4129005472$

H $1.2045581107 \quad 2.3649898997 \quad-0.6636071035$

H $\quad 0.3712253429 \quad 1.81328484610 .8093761265$

Frequencies

28.456

200.454

601.640

648.002

746.390

799.790

1233.466

1434.758

1446.680

3061.142

3146.941

3174.034

\section{$\mathrm{H}_{3} \mathrm{CSSi}$}

$\begin{array}{lrrr}\text { C } & -1.8311634692 & 0.2262129791 & 0.2401343951 \\ \text { Si } & 1.2847895101 & 0.7324340425 & 0.3716915006 \\ \text { H } & -1.5104170895 & 0.1778200986 & 1.2776627094 \\ \text { H } & -2.2712699269 & -0.7213070719 & -0.0593963275 \\ \text { S } & -0.3974226854 & 0.5682218748 & -0.8452514948 \\ \text { H } & -2.5591441481 & 1.0228578845 & 0.1107956769\end{array}$

Frequencies

91.185

216.923

537.685

690.499

941.809

974.020

1339.821

1471.766

1480.365

3078.111

3175.993

3176.393

\section{$\mathrm{H}_{2} \mathrm{CSiSH}$}


Si $\quad-1.04456202521 .7530735297 \quad-0.9807337388$

$\begin{array}{lllll}\text { C } & 0.2413969917 & 2.6328136230 & 0.0017374659\end{array}$

$\begin{array}{llll}\text { S } & -2.7987231043 & 2.7995066603 & -0.2966019905\end{array}$

$\mathrm{H} \quad 0.0884899935 \quad 3.0436510667 \quad 0.9955430419$

H $\quad 1.27836558662 .6460468081 \quad-0.3191422582$

H $\quad-2.2362652823 \quad 3.7285089322 \quad 0.4877366297$

Frequencies

210.468

262.133

357.417

484.335

628.457

678.220

714.842

778.981

1403.638

2712.465

3121.218

3213.255

\section{$\mathrm{H}_{2} \mathrm{CSSiH}$}

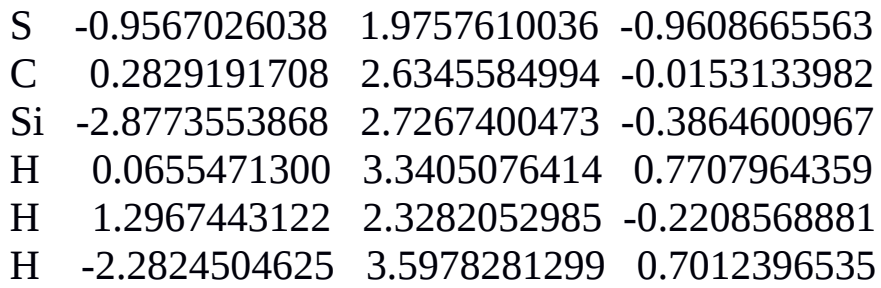

Frequencies

254.375

265.798

374.468

492.900

549.573

789.068

818.251

947.315

1412.589

2073.627

3170.620

3300.879 


\section{Intermediates}

\section{i1: $\mathrm{H}_{3} \mathrm{CSHSi}$}

S $\quad 0.7535961169-0.1476115477-0.5173300205$

C $\quad-0.9562773727 \quad 0.1198347642 \quad 0.0329041416$

H $\quad-1.3128232823 \quad 0.9755020966 \quad-0.5344925859$

H $\quad-0.9596138790 \quad 0.3617587404 \quad 1.0920844411$

Si $\quad 1.9349681829 \quad 1.6840828295 \quad 0.3313963364$

H $\quad-1.5628324519-0.7556180383-0.1850482300$

H $\quad 1.0196708961 \quad-1.1677568348 \quad 0.3166149375$

Frequencies

159.376

171.928

291.118

531.924

700.609

790.534

977.839

1086.982

1349.374

1466.167

1473.789

2646.142

3081.432

3184.048

3186.853

\section{i2: $\mathrm{H}_{3} \mathrm{CSSiH}$}

C $\quad-1.7971468245 \quad 0.1665999445 \quad 0.2578597815$

Si $1.0906267164 \quad 1.2724759431 \quad 0.2543602640$

H $\quad-1.6161079716 \quad-0.1763601524 \quad 1.2722259916$

H $\quad 1.15488851912 .6988159624 \quad-0.1525096098$

$\mathrm{H} \quad-2.5411101317-0.4716157687 \quad-0.2141035755$

S $\quad-0.2721496092 \quad 0.0210067579-0.7395683877$

H $\quad-2.1487399884 \quad 1.1941330831 \quad 0.2648623859$

Frequencies

108.135

198.960 
294.803

528.155

631.713

702.876

966.094

974.731

1345.001

1475.652

1479.910

2182.496

3082.302

3172.539

3182.960

\section{i3: $\mathrm{H}_{3} \mathrm{CSSiH}$}

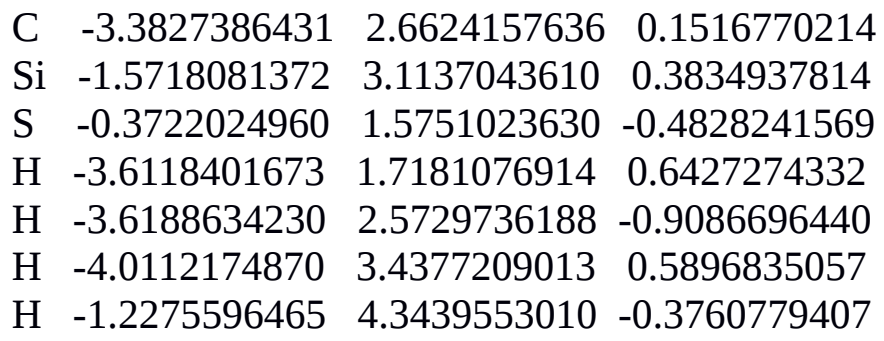

Frequencies

136.780

163.770

488.990

531.621

599.910

695.913

789.148

870.727

1264.975

1451.055

1457.622

2195.953

3064.181

3148.814

3156.815

\section{i4: $\mathrm{H}_{3} \mathrm{CSiHS}$}

Si $\quad-1.2458788518 \quad 2.3231009761 \quad-0.9781839766$

C $\quad 0.3538990350 \quad 2.5314614087 \quad 0.0048513910$ 


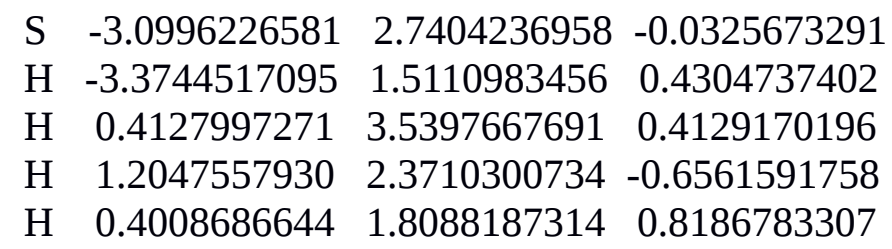

Frequencies

93.421

177.183

272.428

489.717

687.808

723.740

785.220

795.956

1250.409

1447.944

1451.854

2677.464

3061.699

3148.885

3154.707

\section{Transition states}
i1 - i2
S $\quad 0.7481780063-0.0822070340-0.8525894498$
$\begin{array}{lllll}\text { C } & -0.8492021348 & 0.1082606578 & -0.0125276989\end{array}$
H $\quad-1.34627523120 .9896038306 \quad-0.4089109885$
$\begin{array}{llll}\mathrm{H} & -0.6724768830 & 0.2433247565 & 1.0530675469\end{array}$
$\begin{array}{lllll}\text { Si } & 2.0423494127 & 1.5548214095 & 0.2135083332\end{array}$
H $-1.4506121282-0.7801925322-0.1864736118$
$\begin{array}{llll}\text { H } & 1.6013989781 & -0.1464216981 & 0.4181428489\end{array}$

Frequencies

$623.663 \mathrm{i}$

113.981

182.596

409.484

679.399

717.074

974.354 
\begin{tabular}{llll}
982.807 & & \\
1348.403 & & \\
1465.433 & & \\
1471.919 & & \\
1484.081 & & \\
3074.159 & & \\
3165.467 & & \\
3179.486 & & \\
i2 $-\mathbf{i 4}$ & & \\
\multicolumn{4}{c}{$\mathrm{C}$} \\
$\mathrm{C}$ & -1.5301634034 & -0.0552895284 & 0.1285954464 \\
$\mathrm{Si}$ & 0.4902640488 & 0.1658458420 & -1.0038804565 \\
$\mathrm{~S}$ & 0.4293183823 & -0.3786115151 & 1.0086322597 \\
$\mathrm{H}$ & -1.7958080293 & 0.9942789921 & 0.1373633933 \\
$\mathrm{H}$ & -1.9530861682 & -0.5639735863 & 0.9868776101 \\
$\mathrm{H}$ & -1.8758576940 & -0.5600036796 & -0.7703438002 \\
$\mathrm{H}$ & 0.2012321238 & -0.8413371546 & -2.0560213327
\end{tabular}

Frequencies

$1153.918 \mathrm{i}$

152.351

359.306

395.345

582.590

650.456

780.006

819.176

1242.002

1439.121

1481.042

2172.933

3089.882

3168.599

3221.493

i3 - i4

Si $\quad-0.5242511057 \quad 0.0746288215 \quad-0.4976388744$

C $\quad 0.6087369755 \quad 0.0071929366-2.0042215073$

$\begin{array}{lllll}\text { S } & 0.2827566741 & 0.0215922105 & 1.4399347849\end{array}$

$\mathrm{H} \quad-0.2481535695 \quad-1.1916203276 \quad 0.5579910894$

$\mathrm{H} \quad 1.0341165470 \quad 0.9930479366-2.1904494097$

$\mathrm{H} \quad 0.0460899998 \quad-0.3045415080-2.8826754995$

H $\quad \begin{array}{llll}1.4132532842 & -0.7029903520 & -1.8188214101\end{array}$ 
Frequencies

$1208.268 \mathrm{i}$

103.067

181.806

443.086

537.088

698.386

776.514

787.555

1248.775

1445.029

1452.001

1674.260

3064.349

3151.854

3162.567

i1 - p3

C $\quad-0.7194774720 \quad 0.0267300161 \quad 2.2910587295$

H $\quad-1.4761812181-0.7324624505 \quad 2.1639155760$

H $\quad 0.0736284538-0.1903587240 \quad 2.9921544956$

H $\quad-1.0432497535 \quad 1.0542980628 \quad 2.2151031599$

S $\quad 0.4742360782 \quad-0.2377614662 \quad 0.1966917599$

H $\quad \begin{array}{llll}1.1973049861 & 0.8172951249 & 0.6078055140\end{array}$

Si $-0.4122244745 \quad 0.5119183768$-1.5954806648

Frequencies

394.822 i

75.918

95.622

419.633

460.670

537.167

583.356

693.979

895.558

1422.864

1428.581

2651.294

3116.926

3280.378

3286.219 


$$
\begin{array}{lccc}
\mathbf{i} 2-\mathbf{p} 1 & & \\
& & & \\
\text { C } & 0.6523314477 & -0.0111790027 & -2.4168348591 \\
\text { Si } & 0.1285160059 & 0.1084339313 & 1.4128391872 \\
\text { H } & -0.1111659228 & -0.3531614760 & -3.0965671594 \\
\text { H } & 1.0680026049 & -0.7146165498 & 2.2376055716 \\
\text { H } & 1.5053219784 & -0.6454547594 & -2.2407367647 \\
\text { S } & -0.6689207316 & -0.6085433644 & -0.2293891769 \\
\text { H } & 0.7526867975 & 1.0488562510 & -2.2522248587
\end{array}
$$

Frequencies

257.689 i

46.325

103.239

297.489

423.840

439.544

633.878

675.983

759.672

1420.035

1421.084

2110.037

3137.621

3309.675

3313.448

$$
\begin{array}{lrrr}
\mathbf{i} \mathbf{1}-\mathbf{p} \mathbf{4} & & \\
& & & \\
\mathrm{C} & -0.8514941561 & 0.0057960073 & 1.6196638831 \\
\mathrm{H} & -0.4884599523 & 0.5996148041 & 2.4551046565 \\
\mathrm{H} & -1.7071132737 & 0.4906773293 & 1.1566936162 \\
\mathrm{H} & -1.1225604185 & -0.9889408194 & 1.9622120958 \\
\mathrm{~S} & 0.5072723247 & -0.1526533034 & 0.4052042039 \\
\mathrm{H} & 1.5385644267 & 1.4823897055 & 1.4598152027 \\
\text { Si } & -0.2779194008 & 0.3602617665 & -1.4713452883
\end{array}
$$

Frequencies

458.001 i

76.782

185.691

218.219

336.252 
522.070

691.170

950.418

978.393

1340.534

1472.017

1477.165

3078.206

3175.065

3180.812

i3 - p2

Si $\quad-0.5429894632-0.0155967501-0.4888451895$

C $\quad 0.5194410958-0.0170592525-2.0445820662$

S $\quad 0.2068322020 \quad 0.1225332353 \quad 1.3113741480$

H $\quad 0.2881308659-2.0150159796 \quad 2.3472828756$

H $\quad 1.1873447863 \quad 0.8438089250-2.0115142810$

H $\quad-0.0801524271 \quad 0.0100821807 \quad-2.9510387368$

H $1.1229344874-0.9262028526-2.0344329433$

Frequencies

389.096 i

33.377

110.409

177.238

204.162

603.703

662.319

745.716

799.239

1236.102

1435.082

1446.453

3061.168

3147.114

3173.541 